\title{
Diffraction Plane Dependence of Micro Residual Stresses in Uniaxially Extended Carbon Steels
}

\author{
T. Hanabusa ${ }^{1^{\star}}$, A. Shiro ${ }^{2}$, M. Refai $^{3}$ and M. Nishida ${ }^{4}$ \\ ${ }^{I}$ Faculty of Engineering, The University of Tokushima, Minami-Josanjima, Tokushima, Japan \\ ${ }^{2}$ Graduate School of Advanced Technology and Science, The University of Tokushima, Minami- Josanjima, Tokushima, Japan \\ ${ }^{3}$ Center for Technology of Nuclear Industry Materials, National Nuclear Energy Agency \\ Puspiptek, Serpong, Tangerang 15314, Indonesia \\ ${ }^{4}$ Kobe-Kosen, Gakuen-Higashi, Kobe, Japan
}

\section{ARTICLE INFO}

Article history:

Received 30 September 2010

Received in Revised form 28 October 2010

Accepted 1 November 2010

Keywords:

Carbon steel

Micro stress

Neutron diffraction

Residual stresses

\begin{abstract}
A B S T R A C T
In the stress measurement using X-ray or neutron diffraction, an elastic anisotropy as well as a plastic anisotropy of crystal must be carefully considered. In the X-ray and neutron diffraction stress measurement for polycrystalline materials, a particular $\{\mathrm{hkl}\}$ plane is used in measuring lattice strains. The dependence of an X-ray elastic constant on a diffraction plane is a typical example caused by an elastic anisotropy of the crystal. The yield strength and the work hardening rate of a single crystal depend on a crystallographic direction of the crystal. The difference in the yield strength and the work hardening rate relating to the crystallographic direction develops different residual stresses measured on each $\{\mathrm{hkl}\}$ diffraction after plastic deformation of a polycrystalline material. The present paper describes the result of the neutron stress measurement on uniaxially extended low and middle carbon steels. A tri-axial residual stress state developed in the extended specimens was measured on different kind of $\{\mathrm{hkl}\}$ diffraction plane. The measurement on the $\{110\},\{200\}$ and $\{211\}$ diffraction showed that residual stresses increased with increasing the plastic elongation and the residual stresses on $\{110\}$ were compressive, $\{200\}$ were tensile and those on $\{211\}$ were the middle of the former two planes.
\end{abstract}

(C) 2010 Atom Indonesia. All rights reserved

\section{INTRODUCTION}

In the stress measurement by diffraction, there are strict problems according to the kind of $\{h k l\}$ plane. The first one is the elastic anisotropy [1]. This is relating to the different of elastic constant according to $\{\mathrm{hkl}\}$ plane. The second one is the plastic anisotropy resulted by difference in the plastic property according to the crystal orientation [2]. Furthermore, if the material contains more than two phases, phase stress comes to be another problem called as a phase stress. Typical examples are the case of residual stresses in a high carbon steel containing ferrite and cementite phases [3] and $\alpha+\beta$ brass [4]. This is also due to the difference in the ability of plasticity among consisting phases.

The objectives of the present study are to investigate the plastic anisotropy of micro residual stress development and also to investigate the dependence of carbon content on the residual stress

\footnotetext{
* Corresponding author.

E-mail address: hanabusa@ip.tokushima-u.ac.jp (T. Hanabusa)
}

development. In order to investigate these things, a tri-axial stress measurement was carried out on different diffraction planes by neutron diffraction.

\section{EXPERIMENTAL METHODS}

The specimens used in this experiment were a low carbon steel SS400 and a medium carbon steel S45C, symbolized by Japanese Industrial Standard. The carbon content of each material is below $0.2 \%$ and $0.45 \%$, respectively. Two types of tensile specimen were prepared. The first one was for SS400 and S45C_a and had a square cross section with $12 \mathrm{~mm} \times 12 \mathrm{~mm}$ and a gage length of $60 \mathrm{~mm}$. The second one was for $\mathrm{S} 45 \mathrm{C} \_\mathrm{b}$ having a circular cross section with a diameter of $15 \mathrm{~mm}$ and a gage length of $60 \mathrm{~mm}$.

In order to relieve initial residual stress, they were annealed in a vacuum furnace at $700{ }^{\circ} \mathrm{C}$ or 600. Therefore, the micro structure is ferrite and ferrite/pearlite in each material. After annealing treatment, SS400 was extended to about 5, 10 and 
$20 \%$, and $\mathrm{S} 45 \mathrm{C}$ was about $1,5,10$ and $15 \%$ as listed in Table 1.

Table 1. Materials of specimen and pre-deformation in tension.

\begin{tabular}{|c|c|c|c|}
\hline Material & SS400 & S45C (a) & S45C (b) \\
\hline Carbon content, \% & $<0.2$ & 0.45 & 0.45 \\
\hline Cross section, $\mathrm{mm} \times \mathrm{mm}$ & $12 \times 12$ & $12 \times 12$ & ( $15 \mathrm{~mm}$ in dia.) \\
\hline $\begin{array}{l}\text { Temperature of stress } \\
\text { relief treatment, deg in C }\end{array}$ & 700 & 700 & 600 \\
\hline Micro structure & Ferrite & $\begin{array}{c}\text { Ferrite }+ \\
\text { pearlite }\end{array}$ & $\begin{array}{l}\text { Ferrite + } \\
\text { pearlite }\end{array}$ \\
\hline $\begin{array}{l}\text { Pre-deformation in } \\
\text { tension, \% }\end{array}$ & $5,13,21$ & $\begin{array}{l}1,4,8 \\
15\end{array}$ & $1,5,10,15$ \\
\hline
\end{tabular}

Neutron stress measurement was made by the residual stress measurement system in Neutron Scattering Laboratory of the Center for Technology of Nuclear Industrial Materials of National Nuclear Energy Agency (BATAN), Indonesia. Fig. 1 shows the experimental set up for stress measurement. The wave length of neutron beam was $0.183404 \mathrm{~nm}$. In order to restrict the cross section of the incident beam, $3 \mathrm{~mm} \times 3 \mathrm{~mm}$ incident slit was used for $\mathrm{SS} 400$ and S45 C a and $5 \mathrm{~mm} \times 5 \mathrm{~mm}$ incident slit for $\mathrm{S} 45 \mathrm{C} \mathrm{b}$ and in order to determining the diffraction angle, $3 \mathrm{~mm} \times 10 \mathrm{~mm}$ for SS400 and S45 C_a and $5 \mathrm{~mm} \times 5 \mathrm{~mm}$ was used for S45 C_b.

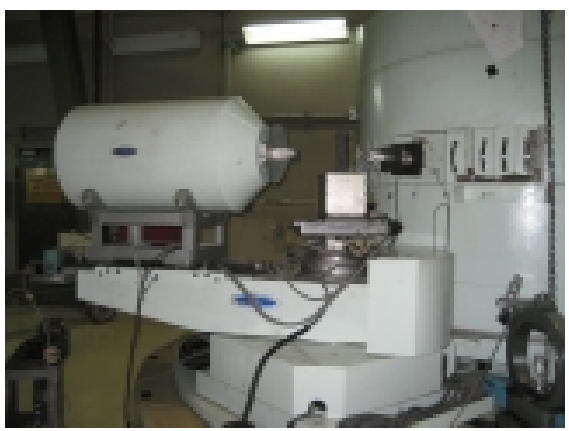

(a)

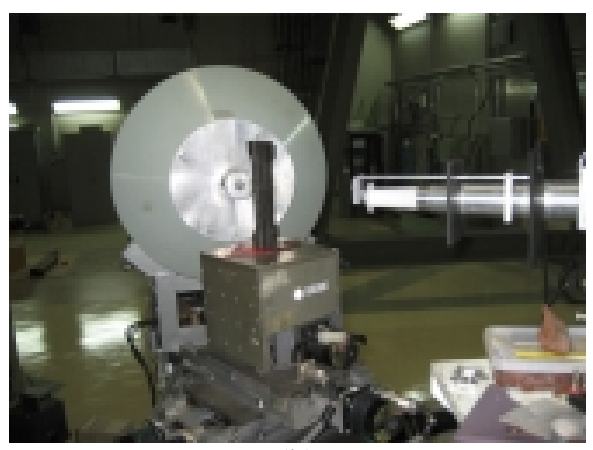

(b)

Fig. 1. Experimental set up for neutron stress measurement: (a) view of neutron diffractometer, (b) setting of specimen on the sample table.
After the specimen was extended up to a strain level as mentioned above, residual stresses were measured at the middle point of the tensile specimen. Table 2 shows the experimental conditions for the neutron stress measurement.

Table 2. Neutron stress measurement system.

\begin{tabular}{ll}
\hline Facility & $\begin{array}{l}\text { Neutron Scattering Laboratory of Center } \\
\text { for Technology of Nuclear Industrial } \\
\text { Materials of National Nuclear Energy } \\
\text { Agency (BATAN), Indonesia }\end{array}$ \\
\hline $\begin{array}{l}\text { Equipment } \\
\text { Wave length of }\end{array}$ & $\begin{array}{l}0.183404 \mathrm{~nm} \\
\text { neutron beam }\end{array}$ \\
Incident slit & $3 \mathrm{~mm} \times 3 \mathrm{~mm}$ for SS400 and S45C (a) \\
& $5 \mathrm{~mm} \times 5 \mathrm{~mm}$ for S45C (b) \\
Receiving slit & $3 \mathrm{~mm} \times 10 \mathrm{~mm}$ for SS400 and S45C (a) \\
& $5 \mathrm{~mm} \times 5 \mathrm{~mm}$ for S45C (b) \\
\hline
\end{tabular}

\section{Evaluation for neutron stress measurement}

The coordinate system was determined as $\mathrm{x}_{1}$ (directed along the specimen axis), $x_{2}$ (in the transverse direction) and $\mathrm{x}_{3}$ (along the normal of the specimen surface). Stress evaluation was made by the three dimensional Hooke's law as shown in the following equation. The necessary $\mathrm{d}_{0}$ value for evaluating strain was determined from the annealed specimen.

$$
\left\{\begin{array}{l}
\varepsilon_{1}=\frac{1}{E}\left\{\sigma_{1}-v\left(\sigma_{2}+\sigma_{3}\right)\right\} \\
\varepsilon_{2}=\frac{1}{E}\left\{\sigma_{2}-v\left(\sigma_{1}+\sigma_{3}\right)\right\} \\
\varepsilon_{3}=\frac{1}{E}\left\{\sigma_{3}-v\left(\sigma_{1}+\sigma_{2}\right)\right\}
\end{array}\right.
$$

In order to investigate the dependence of diffraction plane on residual stresses, three types of diffraction, $\{110\},\{200\}$ and $\{211\}$, were used in this experiment. According to equation (1), the diffraction angles were measured in three principal directions. $2 \theta$ angles were converted to strains by Bragg's law with the help of $2 \theta_{0}$ value determined using annealed specimen.

As the diffraction elastic constants, the values calculated by the Kröner model were used in the stress evaluation and tabulated in Table 3.

Table 3. Diffraction elastic constants for three types of diffraction plane.

\begin{tabular}{ccc}
\hline Diffraction plane & $E_{\text {diffraction }}(\mathrm{GPa})$ & $v_{\text {diffraction }}$ \\
\hline$\{110\}$ & 224 & 0.28 \\
$\{200\}$ & 174 & 0.33 \\
$\{211\}$ & 224 & 0.28 \\
\hline
\end{tabular}




\section{RESULTS AND DISCUSSION}

Figures 2 and 3 show the typical examples of three kind diffraction patterns of $\{110),\{200\}$ and $\{211\}$ observed from the low carbon steel and the middle carbon steel, respectively. Fairly good intensity profiles were obtained so as to determine the peak positions by the Gaussian fitting method.

Figure 4 shows the results of residual stresses in low carbon steel specimen having a squared cross section. Due to the plastic deformation, tri-axial residual stresses were developed in the specimen. The residual stress increased with increasing the plastic deformation, in particular, in the early stage of the plastic deformation. No systematic difference among the stress components of $\sigma_{1}, \sigma_{2}$ and $\sigma_{3}$ can be seen. However, there was a clear difference in the stresses measured from different diffraction plane. The plastic anisotropy in the residual stress was observed, i.e., the stresses measured from $\{200\}$ plane showed large tensile and those of $\{110\}$ were small compressive state in all three principal directions and the stresses observed in $\{211\}$ plane were the middle value of $\{200\}$ and $\{110\}$, therefore, they were small compression or small tension.

Figure 5 shows the results observed in the samples of the medium carbon steel having a squared cross section. The result of $\sigma_{1}$ is only the exception, but other two results show the same tendency with the results of low carbon steel. Namely, residual stresses measured by $\{200\}$ plane were tensile excepting the case of $\sigma_{1}$ and those by $\{110\}$ were compressive. Stresses measured by $\{211\}$ showed small values as in the case of the low carbon steel, SS400.

From a rough observation, we can see the residual stresses developed in middle carbon steel are shifted by a small amount to compressive side in comparison with the low carbon steel.

Considering the square cross section of the gage portion of tensile specimen, $\sigma_{2}$ and $\sigma_{3}$ should be the same with each other. However, these stress components were different both in the cases of low carbon steel and mild carbon steel. There are also errors in measuring residual strains by neutron diffraction but main reason may be the restriction of deformation by the non-symmetrical mode of grasping a specimen in tensile deformation.

In order to observe the effect of the shape of cross section of the gage portion, specimens having a circular cross section were prepared. Fig. 6 shows the results of residual stress measurement on S45C (b). Fig. 6 (a), (b) and (c) are the results measured by $\{110\},\{200\}$ and $\{211\}$ plane, respectively. In this case, $\sigma_{2}$ was almost equal to $\sigma_{3}$ in this case.
Figure 6 (d) is the summary of the axial residual stress component, $\sigma_{1}$, measured on three diffraction planes. Large compressive stresses in $\{110\}$ plane and small compressive stresses in $\{200\}$ plane were observed. Stresses in $\{211\}$ expressed middle values between those of $\{110\}$ and $\{200\}$.
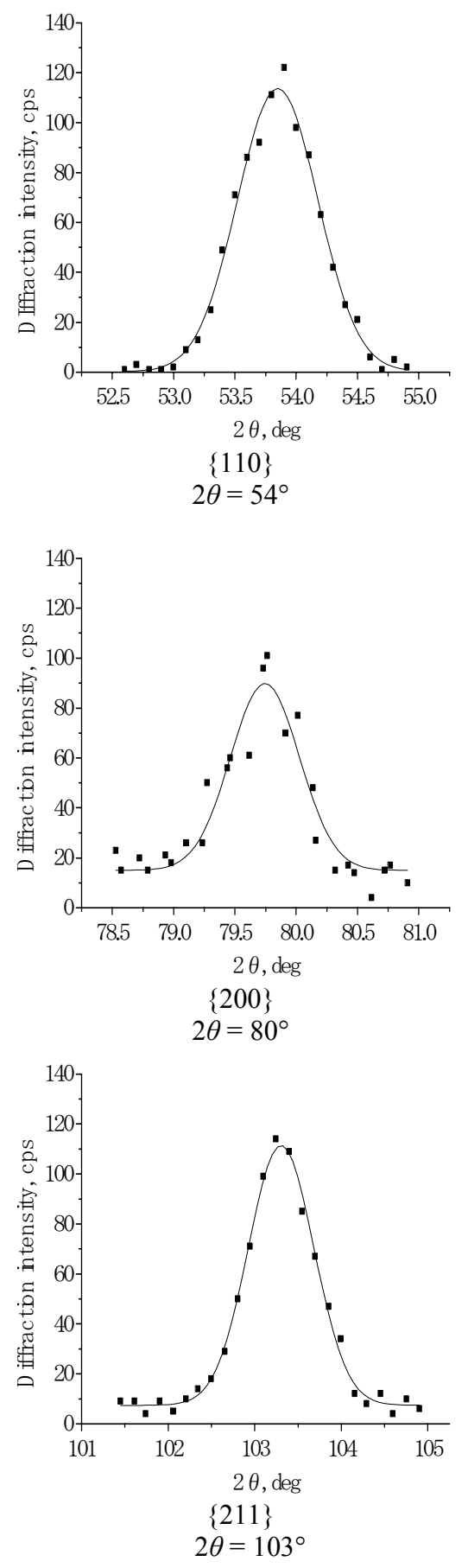

Fig. 2. Neutron diffraction patterns of $\{110\},\{200\}$ and $\{211\}$ observed from low carbon steel. 

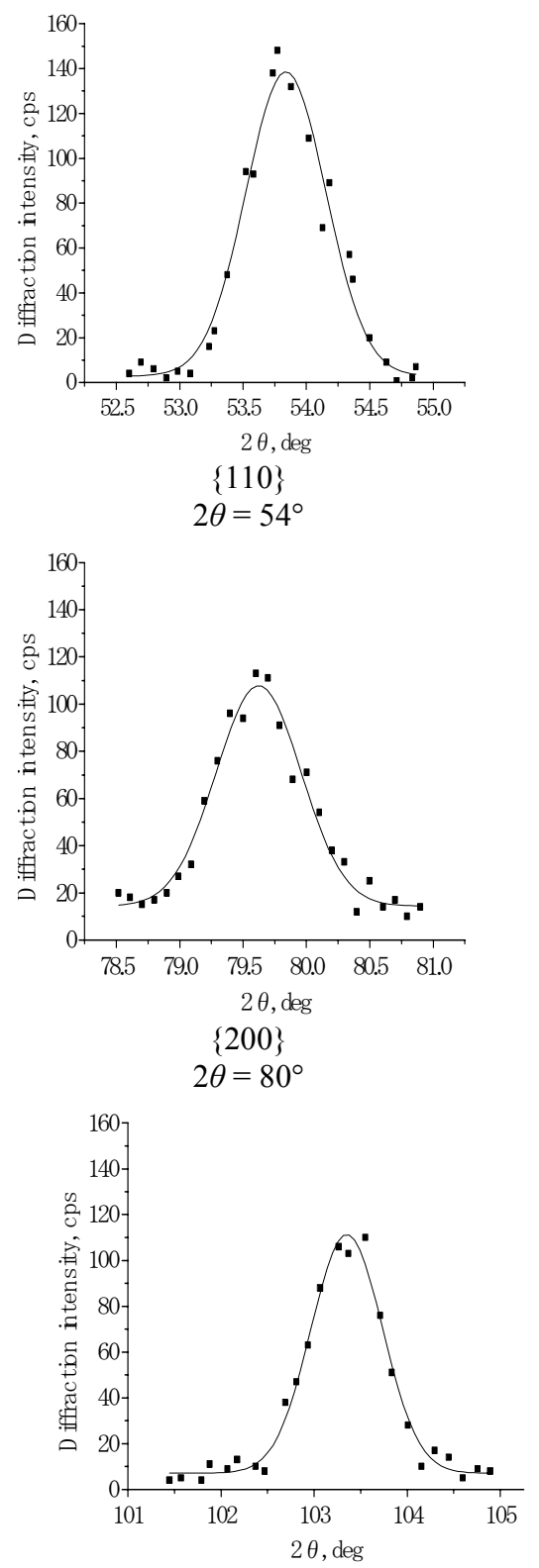

$\{211\}$

$2 \theta=103^{\circ}$

Fig. 3. Neutron diffraction patterns of $\{110\},\{200\}$ and $\{211\}$ observed from middle carbon steel.

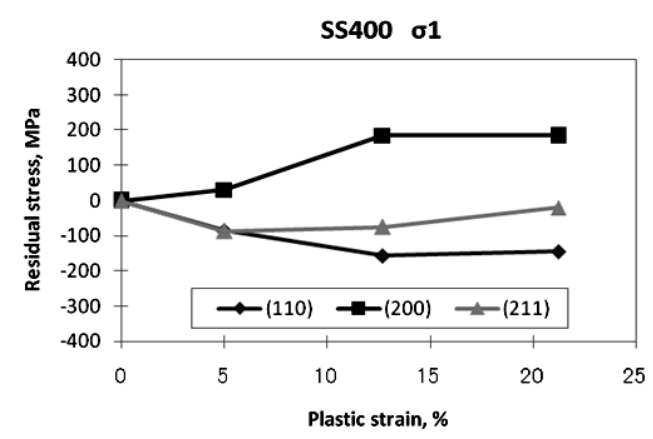

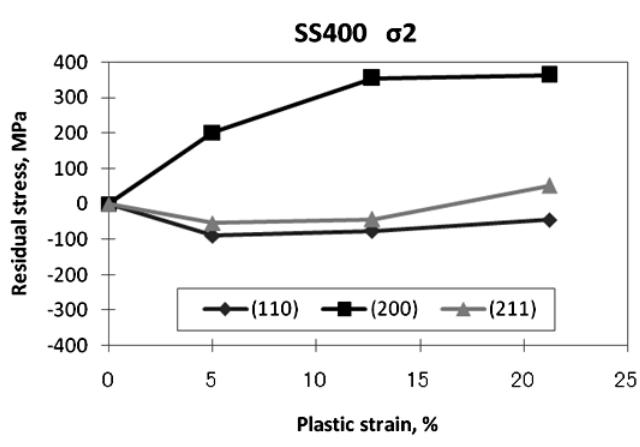

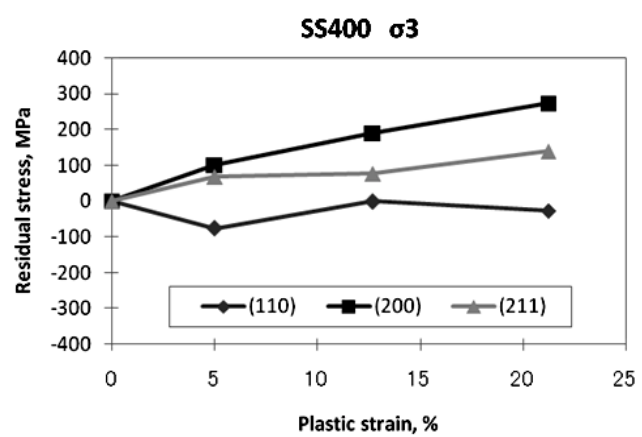

Fig. 4. Residual stress development in low carbon steel, SS400.
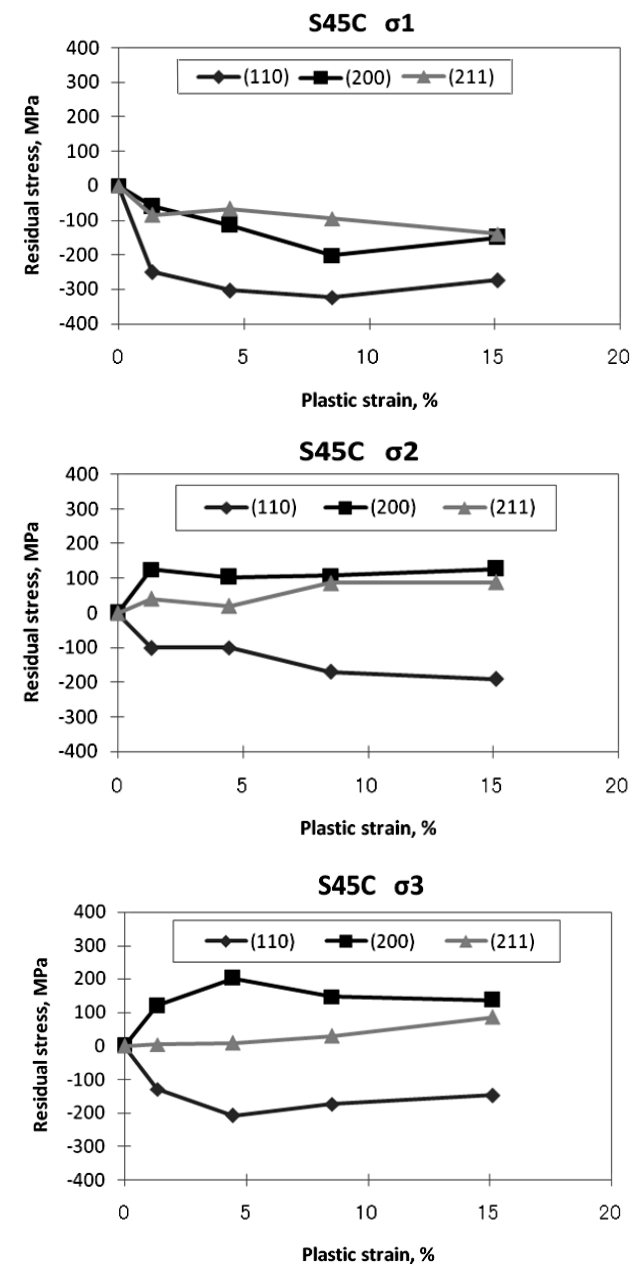

Fig. 5. Residual stress development in medium carbon steel, $\mathrm{S} 45 \mathrm{C}$ a. 
(a) $\mathrm{S45C}(110)$

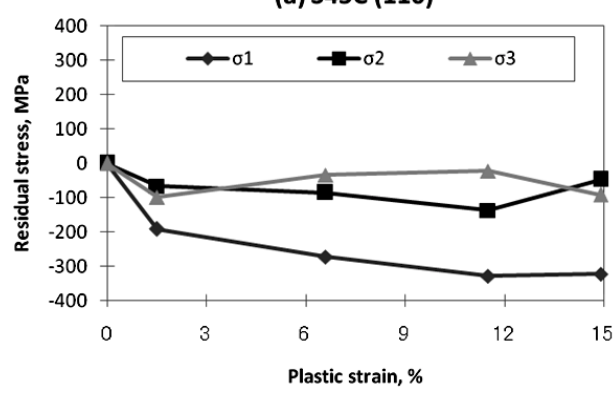

(b) $545 C$ (200)

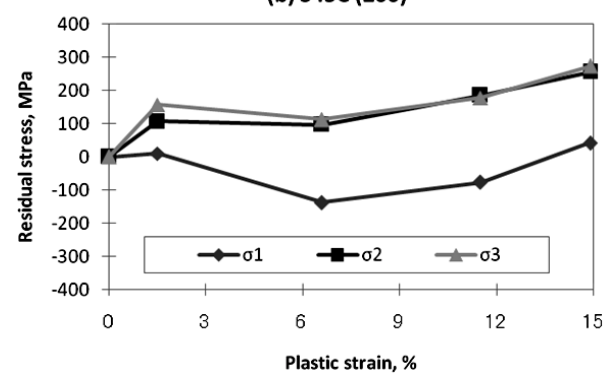

(c) $\mathrm{S45C}(211)$

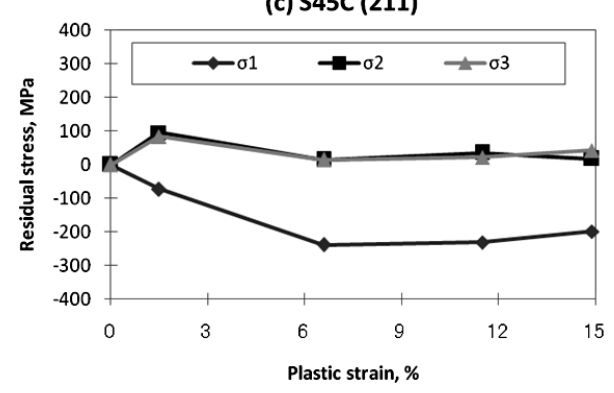

(d) $\mathrm{S} 45 \mathrm{C} \sigma 1$

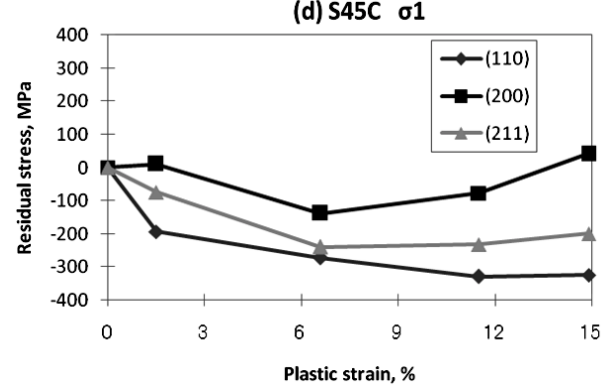

Fig. 6. Results of residual stress measurement on S45C_b specimen having a circular cross section.

The present investigation includes two problems. The first one is the dependence of diffraction plane on residual stress development and the second one is the dependence of phase stress. The first one includes two meanings: an elastic anisotropy and a plastic anisotropy. The elastic anisotropy is due to the difference of the elastic property of single crystal. If we consider a polycrystalline aggregate with a random orientation of the constituent crystals, the elastic property is homogeneous in every orientation of the bulk material instead each crystal has an elastic anisotropy. In the X-ray stress measurement and the neutron stress measurement, a particular $\{\mathrm{hkl}\}$ plane is used to measure lattice strains. Therefore, the dependence of $\{\mathrm{hkl}\}$ plane develops in the stress measurement. The plastic anisotropy is caused by the slip behavior in a crystal. The slip in a crystal occurs on a slip system with the combination of particular crystal planes and directions. In the case of $\alpha$-iron, the slip systems are $\{110\}[111]$, $\{112\}[111]$ and $\{123\}[111]$. The first one is the most preferable slip system. Because of the above reason, the plastic properties of a single crystal depend on an orientation of the crystal against the deformation axis. Table 4 shows the work hardening rate, which is estimated from the figure of the paper in reference [5].

Table 4. Dependence of crystal orientation of $\alpha$-iron single crystal on work hardening rate and residual stress.

\begin{tabular}{lccc}
\hline & $\begin{array}{c}\text { Crystal } \\
\text { orientation }\end{array}$ & $\begin{array}{c}\mathrm{d} \tau / \mathrm{d} \gamma \text { at } 0.2 \\
\left(\mathrm{~N} / \mathrm{mm}^{2}\right)\end{array}$ & $\begin{array}{c}\text { Measured residual } \\
\text { stress }\end{array}$ \\
\hline \multirow{2}{*}{$\begin{array}{l}\alpha \text {-Fe single } \\
\text { crystal }\end{array}$} & {$[100]$} & 105 & $\{200\}$, tensile \\
\cline { 2 - 4 } & {$[112]$} & 22 & $\{211\}$, small \\
\cline { 2 - 4 } & {$[110]$} & 29 & $\{110\}$, compressive \\
\hline
\end{tabular}

Generally, the development of micro residual stresses can be explained schematically by Fig. 7 . Let us consider that the material is consisted with hard region and soft region. In this figure, a different yield stress and a different work hardening rate are shown by two lines, each indicating hard and soft regions. As shown in the figure, if the applied stress is perfectly relieved followed by some plastic extension, tensile residual stress develops in a hard region and compressive stress in a soft region. Residual stress developed after plastic extension depends on both yield point and work hardening rate. The dependence of residual stress on a particular $\{\mathrm{hkl}\}$ plane of the stress measurement may be explained by the difference of the work hardening rate of the crystal orientation.

In a case of two-phase material, the situation is simpler. A steel specimen contains a cementite phase and a ferrite phase. It is clear that the cementite phase is hard and a ferrite phase is soft. Therefore, tensile residual stress develops in the cementite phase and compressive stress develops in the ferrite phase. One of the authors has shown in the X-ray stress measurement that tensile residual stresses develop in cementite phase and compressive stress in ferrite phase in the high carbon steel specimens extended in tension [4]. The fact that the stresses in the middle carbon steel, S45C, tend to compressive side in compared with those in the low carbon steel, SS400, is due to the existence of the cementite phase in the middle carbon steel. 


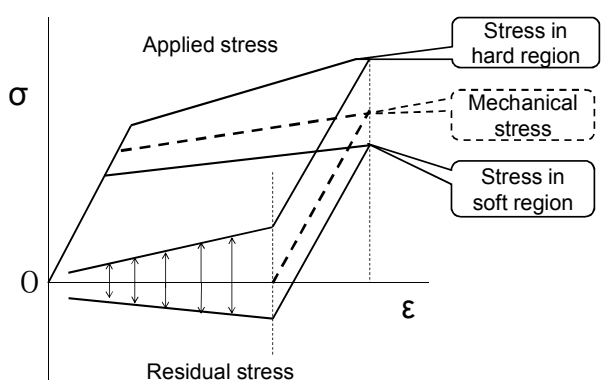

Fig. 7. Schematic model of microstress development.

\section{CONCLUSIONS}

Residual stresses developed in plastically extended low carbon and medium carbon steels were measured by neutron diffraction and micro residual stresses were measured on three kind of $\{\mathrm{hkl}\}$ diffraction planes. The results obtained in the present investigation are as follows: (i) Tri-axial residual state develops in the extended specimen (ii) Residual stresses increase with increasing plastic extension; (iii) The value of residual stresses depends on the diffraction plane. $\{200\}$ : tensile residual stress, $\{211\}$ : middle value between $\{200\}$ and $\{110\},\{110\}$ : compressive residual stress; (iv) Residual stresses in medium carbon steel tend to move in compressive side in compared with those in low carbon steel.

\section{REFERENCES}

1. R. Glocker, Materialprüfung mit Röntgenstrahlen, Springer Verlag (1970) 430.

2. F. Bollenrath, V. Hauk and W. Ohly, Naturwiss 51 (1964) 259.

3. T. Hanabusa, J. Fukura and H. Fujiwara, Bulletin of Japan Society of Mechanical Engineers 35 (1969) 237.

4. T. Hanabusa, B. Scholtes and E. Macherauch, Proc. of the Twentieth Japan Congress on Materials Research (1986) 123.

5. B. Jaoul, Plasticity of Metals (Translated by S. Morozumi and K. Funakubo), Maruzen (1969) 233. 